NORDITA-95/7 N

nucl-th/9502003

\title{
S-wave Meson-Nucleon Interactions and the Meson Mass in Nuclear Matter from Chiral Effective Lagrangians
}

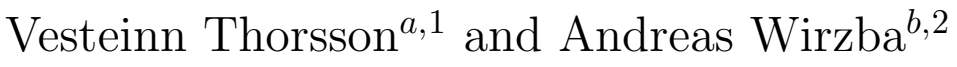 \\ ${ }^{a}$ NORDITA, Blegdamsvej 17, DK-2100 Copenhagen Ø, Denmark \\ ${ }^{b}$ Institut für Kernphysik, Technische Hochschule Darmstadt, \\ Schloßgartenstraße 9, D-64289 Darmstadt, Germany
}

\begin{abstract}
Chiral effective lagrangians may differ in their prediction of meson-nucleon scattering amplitudes off-meson-mass-shell, but must yield identical S-matrix elements. We argue that the effective meson mass in nuclear matter obtained from chiral effective lagrangians is also unique. Off-mass-shell amplitudes obtained using the PCAC choice of pion field must therefore not be viewed as fundamental constraints on the dynamics, the determination of the effective meson mass in nuclear matter or the possible existence of meson condensates in the ground state of nuclear matter. This hypothesis is borne out by a calculation of the effective mass in two commonly employed formulations of chiral perturbation theory which yield different meson-nucleon scattering amplitudes off-meson-mass-shell.
\end{abstract}

\footnotetext{
${ }^{1}$ Email: thorsson@nordita.dk

${ }^{2}$ Email: wirzba@crunch.ikp.physik.th-darmstadt.de
} 


\section{Introduction}

In recent years, there has been considerable discussion of whether Bose condensates of charged mesons may be found in dense nuclear matter, such as that formed in collisions of heavy nuclei, in cores of collapsing stars, or in the interior of neutron stars. These studies have been motivated by the suggestion of Kaplan and Nelson[1] that attractive S-wave interactions between kaons and nucleons could lower the effective mass of kaons to the extent that kaons could condense in dense neutron star matter, at several times nuclear saturation density. The kaon-nucleon interactions of Ref.[1] are obtained from a flavour $S U(3)_{L} \times S U(3)_{R}$ lagrangian, originally due to Manohar and Georgi[2], but with the inclusion of additional terms in the expansion in powers of $\Lambda^{-1}$, where $\Lambda \simeq 1 \mathrm{GeV}$ is the scale of chiral symmetry breaking. The $\mathrm{S}$-wave attraction is driven by two terms in the lagrangian, one related to the exchange of vector mesons between kaons and nucleons and the other proportional to the kaon-nucleon sigma term, $\Sigma^{K N}$, corresponding to the exchange of a scalar meson. The value of the critical density for kaon condensation in such models varies, due to the large uncertainty for estimates of the sigma term: $\Sigma^{K N} \approx(200-400) \mathrm{MeV}$. In the dense interior of neutron stars, a condensate may be formed if the effective kaon mass is brought down to the value of the charge chemical potential, typically around $200 \mathrm{MeV}$ at the densities under consideration, converting the electrons and muons in beta equilibrium with the matter to kaons. For the range of $\Sigma^{K N}$ quoted above, the critical density lies in the range 3-5 $\rho_{0}$, in terms of the nuclear matter saturation density $\rho_{0}=0.16 \mathrm{fm}^{-3}$ [1, 3, 田.

The formation of a condensate depends crucially on the nature of the kaonnucleon interaction. A number of extensions of the original model[1] have been made. One direction of development has been the inclusion of additional terms in the chiral expansion. The standard chiral counting orders scattering amplitudes as a power series in the characteristic energy-momentum scale, $Q$, with the leading order kaon-nucleon amplitude being $\mathcal{O}\left(Q^{1}\right)$. Brown et al. 5 in included the complete set of terms of the same order as the sigma term, $\mathcal{O}\left(Q^{2}\right)$, and fitted the expansion coefficients to $K N$ scattering lengths. Lee and collaborators 6 have included the next order in chiral perturbation theory, $\mathcal{O}\left(Q^{3}\right)$, which includes one-loop diagrams, and extended the fit to include $\bar{K} N$ scattering amplitudes. Several studies have 
also included contributions difficult to access in chiral perturbation theory, such as resonance contributions [6] and effects from nuclear correlations [6, 7]. Another line of development, suggested by Brown, Koch and Rho 8 has been the application of the ideas of Ref. [1] to S-wave pion condensation. However, the corresponding pion-nucleon sigma term $\Sigma^{\pi N} \simeq 45 \mathrm{MeV}$ is considerably smaller than $\Sigma^{K N}$. Also, interactions mediated by the exchange of vector mesons, $\rho$-meson exchange in the case of pions, and both $\rho$ - and $\omega$-meson exchange in the case of kaons, are generally favourable for S-wave kaon condensation, but not for S-wave pion condensation. More specifically, the pion-proton vector meson exchange potential, is only half as attractive as that for kaons, while the corresponding pion-neutron potential is repulsive, and of the same magnitude as for kaons. The vector mediated pion-nucleon interaction is thus repulsive in neutron-rich matter. The inclusion of additional terms in the chiral expansion, fitted to pion-nucleon scattering lengths, therefore leads to a in-medium pion mass that increases slightly with density. The increase to linear order in density was reemphasized in the work of Delorme, Ericson and Ericson[9].

Recently, several author have claimed that the method used to motivate and describe meson condensation is incorrect 9, 10, 11]. They argued that chiral effective lagrangians are inconsistent with current algebra and PCAC [9, 10] for the following reason: off-meson-mass-shell meson-nucleon scattering amplitudes computed from the lagrangian conventionally employed in the description of meson condensation (i.e. of the Kaplan and Nelson [1] type) are not those obtained using PCAC. Secondly, they claimed that the incorporation of these off-meson-mass-shell amplitudes in the calculation inevitably leads to an effective repulsion which serves to inhibit meson condensation 10, 11.

In this Letter, we argue - in line with well-established theorems 12 - that the Swave meson-nucleon scattering amplitudes obtained off-meson-mass-shell are subject to the choice of meson field, are therefore entirely unphysical, and are thus not to be viewed as constraints on a theory. However, the effective meson mass in nuclear matter behaves as the on-meson-mass-shell scattering amplitude, in that it is independent of the choice made for the meson field. ( The identical conclusion was reached independently in the most recent work of Lee et al. [6], through slightly 
different arguments. ) We argue that this is to be understood as a consequence of a general rule: any physically relevant observable is independent of the choice of meson field variables, as is the case for S-matrix elements[12]. To support this conjecture, using a formulation of chiral perturbation theory for which the canonical meson field $i s$ to be identified with the divergence of the axial vector current, namely that originating in the work of Gasser and Leutwyler[13], we obtain an effective meson mass identical to that found in the traditional treatment, originally due to Kaplan and Nelson[1], in which the meson field is not to be identified with the divergence of the axial vector current.

Below, we discuss how the above ideas apply to the analysis of S-wave pion condensation. We thus sidestep the issue of whether the usual assumptions of smoothness of amplitudes used in conjunction with PCAC, are at all good in the SU(3) sector, which they evidently are not, given the relatively large size of the kinematical region over which the smoothness assumptions are to assumed to hold. However, the conclusions reached are directly applicable to the discussion of S-wave kaon condensation. We consider tree level lagrangians throughout, working to $\mathcal{O}\left(Q^{2}\right)$. We illustrate our results for homogeneous, isotropic, isospin symmetric and spinunpolarized nuclear matter, and evaluate nucleon operators in the mean field approximation, such that the corresponding results hold modulo nuclear correlation corrections. The Letter is organized as follows. In Section 2, we review the kinematics of pion-nucleon scattering and amplitudes obtained from PCAC. We outline two approaches to chiral lagrangians, one originating in the work of Manohar and Georgi[2], and the other in that of Gasser and Leutwyler [13]. We discuss how the derived pion-nucleon scattering amplitudes relate to the PCAC amplitudes. In Section 3 , we show that the effective pion mass obtained in both formalisms give identical results. The section concludes with a discussion of the Gell-Mann-Oakes-Renner relation[14] and a summary of results. 


\section{Off-mass-shell Pion-Nucleon Scattering Amplitudes and Chiral Lagrangians}

Low-energy pion-nucleon scattering was widely discussed in the 60s, when ideas such as current algebra and PCAC were developed. A very convenient choice of kinematical variables in the description of pion-nucleon scattering is the set $\nu, \nu_{B}$, $q^{2}$, and $q^{\prime 2}$, where

$$
\nu=\frac{s-u}{4 m_{N}}=\frac{\left(p+p^{\prime}\right) \cdot\left(q+q^{\prime}\right)}{4 m_{N}} \text { and } \nu_{B}=\frac{t^{2}-q^{2}-q^{2}}{4 m_{N}}=-\frac{q \cdot q^{\prime}}{2 m_{N}},
$$

in terms of the incoming ( outgoing ) pion four-momentum, $q\left(q^{\prime}\right)$ and nucleon momentum $p\left(p^{\prime}\right)$, and the Mandelstam variables $s=(p+q)^{2}, t=\left(q-q^{\prime}\right)^{2}$, $u=\left(p^{\prime}-q\right)^{2}$. In what follows, the nucleon, of mass $m_{N}$ is taken on mass-shell, while the pion, of mass $m_{\pi}$, may be off mass-shell. We consider the isospin even pion nucleon scattering amplitude with the pseudovector pole term subtracted, $\bar{D}^{+}$. In the standard decomposition this amplitude reads 15

$$
\begin{array}{r}
\bar{D}^{+}\left(\nu, \nu_{B}, q^{2}, q^{\prime 2}\right)=A^{+}\left(\nu, \nu_{B}, q^{2}, q^{\prime 2}\right)+\nu B^{+}\left(\nu, \nu_{B}, q^{2}, q^{\prime 2}\right) \\
-\frac{\nu_{B}^{2}}{\nu_{B}^{2}-\nu^{2}} \frac{g^{2}}{m} K\left(q^{2}\right) K\left(q^{\prime 2}\right),
\end{array}
$$

where $\mathrm{g}$ and $K\left(q^{2}\right)$ are, respectively, the $\pi N N$ coupling constant and vertex form factor. The amplitude $\bar{D}^{+}$is closely related to the pion-nucleon sigma commutator,

$$
\begin{aligned}
\sigma_{\pi N}(t) & =\frac{1}{3} \sum_{a=1}^{3}\left\langle P\left(p^{\prime}\right)\left|\left[Q_{5}^{a},\left[Q_{5}^{a}, \mathcal{H}_{\mathrm{SB}}\right]\right]\right| P(p)\right\rangle \\
& =\frac{m_{u}+m_{d}}{2}\left\langle P\left(p^{\prime}\right)|\bar{u} u+\bar{d} d| P(p)\right\rangle,
\end{aligned}
$$

where $|P(p)\rangle$ is the proton state of four-momentum $p$, normalized to $1, Q_{5}^{a}$ is the chiral charge of flavour $a$, and $\mathcal{H}_{\mathrm{SB}}=\left(m_{u}+m_{d}\right)(\bar{u} u+\bar{d} d) / 2+m_{s} \bar{s} s$ is the explicitchiral-symmetry-breaking Hamiltonian density, with $m_{u}, m_{d}$ and $m_{s}$ the masses of the up, down and strange quarks, respectively. There are many choices for the pion field, and the one conventionally used is the PCAC choice,

$$
\pi^{a}=\frac{1}{f_{\pi} m_{\pi}^{2}} \partial^{\mu} A_{\mu}^{a}
$$

where $f_{\pi}=93 \mathrm{MeV}$ is the pion decay constant and $A_{\mu}$ is the axial vector current. Under the assumption of a smoothly varying amplitude $\bar{D}^{+}$, one obtains from Eq.(田) 
the simple expressions at the following special kinematical points 16, 17

$$
\begin{aligned}
\text { Weinberg point } \quad \bar{D}^{+}(0,0,0,0) & =-\frac{1}{f_{\pi}^{2}} \sigma_{\pi N}(0), \\
\text { Adler point } \quad \bar{D}^{+}\left(0,0, m_{\pi}^{2}, 0\right) & =\bar{D}^{+}\left(0,0,0, m_{\pi}^{2}\right)=0, \\
\text { Cheng - Dashen point } \quad \bar{D}^{+}\left(0,0, m_{\pi}^{2}, m_{\pi}^{2}\right) & =+\frac{1}{f_{\pi}^{2}} \sigma_{\pi N}(0)+\mathcal{O}\left(m_{\pi}^{3}\right) .
\end{aligned}
$$

The amplitude in the Weinberg point is repulsive and equal in magnitude to the attractive amplitude in the Cheng-Dashen point. The Cheng-Dashen point is distinguished from the others in that both pions are on mass-shell. The sigma-commutator in Eq.([) is evaluated at $t=0$ instead of $t=2 m_{\pi}^{2}$ (the relevant value for the ChengDashen point) as the difference is of order $m_{\pi}^{3}$, and therefore outside the scope of the present discussion. This will be detailed below.

Chiral perturbation theory was developed as a means to obtain Green functions of QCD via an expansion in powers of of the light quark masses and of the external momenta, assumed to be small in relation to the scale of chiral symmetry breaking $\Lambda \approx 1 \mathrm{GeV}$. Here, we shall discuss the predictions of such theories to second order ( next-to-leading order ) in the characteristic momenta. To illustrate our arguments regarding off-mass-shell amplitudes and the effective meson mass in nuclear matter, we examine two formulations of chiral perturbation theory with baryons. The first of these originates in the formalism of Manohar and Georgi[2], and was employed by Kaplan and Nelson[1] in proposing that kaons could form a condensate. The second formulation originates in that of Gasser and Leutwyler 13, which was extended to include baryons by Gasser, Sainio and Švarc 18. A different approach to the baryon sector, using static baryon fields, was formulated by Jenkins and Manohar 19.

We briefly review the chiral effective lagrangian, $\mathcal{L}_{\mathrm{MG}}$, originating in the formalism of Manohar and Georgi[2], including the next-to-leading order terms in chiral perturbation theory[11, 19, 3]. The lagrangian $\mathcal{L}_{\mathrm{MG}}$ is constructed from a $3 \times 3$ matrix, $\Sigma$, involving the pseudoscalar meson octet, and the spin- $\frac{1}{2}$ baryon octet matrix, $B$. Under $S U(3)_{L} \times S U(3)_{R}$, the field transformations are $\Sigma \rightarrow L \Sigma R^{\dagger}$, and $B \rightarrow h B h^{\dagger}$, where $h$ is defined by the transformation law $\xi \rightarrow L \xi h^{\dagger}=h \xi R^{\dagger}$, and where the field $\xi$ is given by $\xi^{2}=\Sigma$. For the purpose of constructing the lagrangian, the current quark mass matrix, $M=\operatorname{diag}\left(m_{u}, m_{d}, m_{s}\right)$, is assigned the $S U(3)_{L} \times S U(3)_{R}$ transformation of $\Sigma$. The chiral expansion, in powers of $\partial_{\mu} \Sigma / \Lambda$ 
and $M / \Lambda$, where $\Lambda$ is the chiral symmetry breaking scale, $\Lambda \approx 1 \mathrm{GeV}$, is constructed so that individual terms in the expansion are $S U(3)_{L} \times S U(3)_{R}$ invariant. Baryons are treated as static, in the formalism first applied to the chiral expansion by Jenkins and Manohar[19]. With a reduction to $S U(2)_{L} \times S U(2)_{R}$ and the S-wave channel, the $\mathcal{O}\left(Q^{2}\right)$ lagrangian reads[5, 20]

$$
\begin{aligned}
\mathcal{L}_{\mathrm{MG}} & =i \bar{N}(v \cdot \partial) N-\sigma \bar{N} N+\frac{1}{2}\left(\partial_{\mu} \pi\right)^{2}-\frac{1}{2} m_{\pi}^{2} \pi^{2} \\
& +\frac{1}{f_{\pi}^{2}}\left(\frac{1}{2} \sigma \pi^{2}+c_{2}(v \cdot \partial \pi)^{2}+c_{3}\left(\partial_{\mu} \pi\right)^{2}\right) \bar{N} N+\cdots
\end{aligned}
$$

The quantity $v_{\mu}$ is the four-velocity of the nucleon, and and reduces to $v_{\mu}=(1,0,0,0)$ in the rest frame of the nucleon. The pion mass is $m_{\pi}=139 \mathrm{MeV}$, and $f_{\pi}=93$ $\mathrm{MeV}$ is the pion decay constant. The values $m_{\pi}^{2}$ and $f_{\pi}^{2}$ include $\mathcal{O}\left(Q^{2}\right)$ loop corrections to the corresponding quantities at tree level, and are used here for notational convenience, since we neglect corrections to the lagrangian of $\mathcal{O}\left(Q^{3}\right)$ and higher. The constants $\sigma, c_{2}$, and $c_{3}$ are linear in the quark masses and therefore of $\mathcal{O}\left(Q^{2}\right)$. The constant $\sigma$ is to be identified with the sigma term, $\sigma(t=0)$, which also serves to increase the nucleon mass over that in the $\mathrm{SU}(2)$ chiral limit, $m_{N}=m_{0}+\sigma$, where $m_{0} \simeq 890 \mathrm{MeV}$, using $\sigma=45 \mathrm{MeV} 21$. Thus the sign of sigma is fixed to be positive. We do not write down the Weinberg ( vector ) term explicitly in the lagrangian above, as it does not enter into the isospin even scattering length, or in the pion self-energy in symmetric nuclear matter, to be considered in Section 3. From the lagrangian (8), we find the isospin even scattering length, $a_{\pi N}^{+}$,

$$
a_{\pi N}^{+}=\frac{1}{4 \pi f_{\pi}^{2}\left(1+m_{\pi} / m_{N}\right)}\left(2\left(c_{2}+c_{3}\right) m_{\pi}^{2}+\sigma\right)+\mathcal{O}\left(m_{\pi}^{3}\right) .
$$

Empirically, $a_{\pi N}^{+}=-0.0083 m_{\pi}^{-1}$ [22], corresponding to a repulsive interaction. Using $\sigma=45 \mathrm{MeV}$, we find $\left(c_{2}+c_{3}\right) m_{\pi}^{2}=-26 \mathrm{MeV}$. Improved values for the constants can be found by including loop corrections [20]: There are terms of $\mathcal{O}\left(m_{\pi}^{3}\right)$ resulting from finite loop corrections, whereas infinite loop corrections and therefore also the corresponding counterterms first appear at $\mathcal{O}\left(m_{\pi}^{4}\right)$ and $\mathcal{O}\left(m_{\pi}^{4} \ln m_{\pi}\right)$. This is the reason why in nuclear matter all quantities of $\mathcal{O}\left(Q^{2}\right)$ get their first correction already at $\mathcal{O}\left(Q^{3}\right)$ and not at $\mathcal{O}\left(Q^{4}\right)$ as their free-space analogs. The lagrangian(8) gives the amplitude

$$
\bar{D}^{+}=+\frac{1}{f_{\pi}^{2}} \sigma+\mathcal{O}\left(m_{\pi}^{3}\right)
$$


in all three kinematical points discussed above, in apparent contradiction with the PCAC result. This statement has been given various interpretations, for example that chiral perturbation theory is incorrect off mass-shell, or in contradiction with well established extrapolations such as PCAC 99, 10, 11].

With regard to the disagreement between Eq.(10) and Eqs.(5,60), it must be kept in mind that the amplitudes (5.66) are not fundamental, in the following sense. Any operator may be adopted to describe the pion field, provided that it is correctly normalized, and has a nonvanishing matrix element between the pion and the vacuum. Amplitudes obtained off-pion-mass shell are subject to the choice adopted for the pion operator and are not unique. ( However, they are not entirely arbitrary, being subject to constraints such as unitarity and causality. ) This does not pose a problem, since the off-pion-mass-shell amplitudes are not accessible by experiment, and as shown by Coleman, Wess and Zumino 12, S-matrix elements, and therefore measured scattering amplitudes, are independent of the choice adopted for the pion field. The result (10) is therefore simply to be understood in the way that the pion field $\pi$ of $\mathcal{L}_{\mathrm{MG}}$ is not the PCAC one.

One should furthermore note that the amplitudes (10) and (77) agree, to $\mathcal{O}\left(Q^{2}\right)$, as required, since the Cheng-Dashen point has the virtue of being on pion-mass-shell. Although the Cheng-Dashen point is unphysical in the sense that it is outside the kinematical region available to pion-nucleon scattering experiments, the scattering amplitude in the Cheng-Dashen point is unique and related to measured amplitudes via dispersion relations. These involve analytic continuations of (on-pion-mass-shell) amplitudes to unphysical regions - unique by Cauchy's theorem, provided the various poles are properly subtracted. A commonly used approach is to perform double dispersion relations in the variables $(\nu, t)$ [15, 18], the degrees of freedom being reduced by two, on pion-mass-shell. A variation in pion-mass can, of course, not be carried out in any experiment; so the Weinberg and Adler points are unphysical in a very different sense than the Cheng-Dashen point.

To conclude this section, we review the evaluation of off-shell scattering amplitudes in the functional integral formulation of chiral perturbation theory developed by Gasser and Leutwyler 13], which was extended to include nucleons by Gasser, Sainio and Švarc [18]. In this approach, a generating functional for Green functions, 
$Z_{\text {eff }}$, and the corresponding lagrangian, $\mathcal{L}_{\mathrm{GSS}}$, are developed as follows. External sources, $s, p, v_{\mu}$, and $a_{\mu}$, are coupled to the quark fields of the QCD action and assigned chiral $S U(2)_{L} \times S U(2)_{R}$ transformations such that the source-extended action is locally chiral invariant. The fact that the sources are coupled in this way ensures that chiral QCD-Ward identities are satisfied. The generating functional for the low-energy effective theory - transcribed to the hadronic level - now depends on the same external sources, where the corresponding hadronic action is formulated in terms of a $2 \times 2$ dummy field $U$, involving mesons. It is a dummy field as it is integrated over in the generating functional formulation. Similarly, one may introduce nucleons, $N(\bar{N})$ coupled to corresponding external sources, $\bar{\eta}(\eta)$, which transform non-linearly under chiral transformations in order to ensure that the coupling terms are (locally) chiral invariant. One identifies the Ward identities of the low-energy effective theory with those of QCD. Note that in this formalism, the sources $s, p$, $v_{\mu}$, and $a_{\mu}$ are coupled from the start to quark bilinears and are therefore directly associated with $S U(2)_{L} \times S U(2)_{R}$ currents in quark variables. The PCAC prescription thus emerges naturally as the sources are transcribed to the hadronic level. The relevant generating functional is

$$
e^{i Z_{\mathrm{eff}}\left[s, p, v_{\mu}, a_{\mu}, \eta, \bar{\eta}\right]}=\mathcal{N} \int d U d N d \bar{N} e^{i \int d^{4} x\left(\mathcal{L}_{\pi \pi}+\mathcal{L}_{\pi N}+\bar{\eta} N+\bar{N} \eta\right)},
$$

where $\mathcal{N}$ is an overall normalization, $U, N, \bar{N}$ are dummy fields, and the lagrangians $\mathcal{L}_{\pi \pi}$ and $\mathcal{L}_{\pi N}$ are dependent on the sources $s, p, v_{\mu}$ and $a_{\mu}$. In what follows, we shall use the scalar source $s$ to generate the quark mass matrix, $s=\mathcal{M}=\operatorname{diag}\left(m_{u}, m_{d}\right)$, and furthermore retain only the source for asymptotic pions, $p$, but set $v_{\mu}=a_{\mu}=0$ ( unless otherwise specified ). The sources $\bar{\eta}$ and $\eta$ generate one-nucleon in- and out-states. As before, the nucleons are treated in the static fermion formalism[19], in which nucleon loops play no role ( see also Appendix A of Ref.[23] ), and the nucleon determinant may therefore taken to be unity.

The lagrangian entering in the generating functional (11), $\mathcal{L}_{\mathrm{GSS}}=\mathcal{L}_{\pi \pi}+\mathcal{L}_{\pi N}$, is to leading order given by the nucleon kinetic energy term and to subleading order, $\mathcal{O}\left(Q^{2}\right)$, by 18,20

$$
\mathcal{L}_{\pi \pi}^{(2)}=\frac{f_{\pi}^{2}}{4} \operatorname{Tr} \partial_{\mu} U \partial^{\mu} U^{\dagger}+\frac{f_{\pi}^{2}}{4} \operatorname{Tr}\left(U^{\dagger} \chi+\chi^{\dagger} U\right),
$$


and,

$$
\mathcal{L}_{\pi N}^{(2)}=-\frac{\sigma}{4 m_{\pi}^{2}} \bar{N} N \operatorname{Tr}\left(U^{\dagger} \chi+\chi^{\dagger} U\right)+c_{2} \bar{N}(v \cdot u)^{2} N+c_{3} \bar{N}(u \cdot u) N
$$

where $u_{\mu}=i u^{\dagger} \partial_{\mu} U u^{\dagger}, U=u^{2}=\exp \left(i \tau^{a} \pi^{a} / f_{\pi}\right), \chi=2 B(s+i p)$, and $v_{\mu}$ is the four-velocity of the nucleon. Expanding $U$ to second order in $\pi^{a}$, we find

$$
\mathcal{L}_{\mathrm{GSS}}=\mathcal{L}_{\mathrm{MG}}+j^{a} \pi^{a}\left(1-\frac{\sigma \bar{N} N}{f_{\pi}^{2} m_{\pi}^{2}}\right)
$$

The pseudoscalar source is $j^{a}=2 B f_{\pi} p^{a}$, in terms of the original source $p^{a}\left(p=p^{a} \tau^{a}\right)$, and of the "quark condensate" $-2 f_{\pi}^{2} B=-2 f_{\pi}^{2} m_{\pi}^{2} /\left(m_{u}+m_{d}\right)$, as follows from the quadratic expansion. Since Green functions are obtained by taking functional derivatives of the generating functional with respect to the source $j^{a}$, the nontrivial coupling of the source to the pion field in Eq.(14) plays an important role.

Solving the equation of motion for the canonical field in terms of the sources, one easily obtains the Ward identity [18]

$$
\begin{aligned}
i \int d^{4} x & \left\langle N\left(p^{\prime}\right)\left|T P^{a}(x) P^{b}(y)\right| N(p)\right\rangle e^{i q^{\prime} x-i q y} \\
= & \frac{g_{\pi}}{m_{\pi}^{2}-q^{2}} \frac{g_{\pi}}{m_{\pi}^{2}-q^{\prime 2}} T_{\pi N}^{a b}\left(p^{\prime} q^{\prime} ; p q\right)
\end{aligned}
$$

where

$$
T_{\pi N}^{a b}=T_{p v}^{a b}+\left\{\frac{q^{2}+q^{2}-m_{\pi}^{2}}{f_{\pi}^{2} m_{\pi}^{2}} \sigma+2 \frac{c_{2} \omega \omega^{\prime}+c_{3} q \cdot q^{\prime}}{f_{\pi}^{2}}\right\} \delta^{a b}
$$

and

$$
g_{\pi} \delta^{a b}=\left\langle 0\left|P^{a}\right| \pi^{b}\right\rangle=2 B f_{\pi} \delta^{a b},
$$

with $P^{a}=\bar{q} i \gamma_{5} \tau^{a} q$ in terms of quark fields. Equivalently, the amplitude (16) follows from the connected $\pi \pi \bar{N} N$ Green function with amputated nucleon legs, $A_{\pi N}\left(q^{2}, q^{\prime 2}\right)$. The function $A_{\pi N}\left(q^{2}, q^{2}\right)$ is given by the Feynman diagrams of Fig. 1 . Fig. 1a) represents the three contributions having two external pion legs. The relevant vertices follow from the interactions in lagrangian (14) quadratic in pion fields, proportional to $\sigma, c_{1}$ and $c_{2}$. Figs. 1b) and 1c) represent the contributions to $A_{\pi N}$ containing only one external pion leg. These terms have weight $-\sigma /\left(f_{\pi}^{2} m_{\pi}^{2}\right)$ and are due to the coupling of the source $j^{a}$ to the nucleon fields in Eq.(14). The sum of all these contributions is

$$
A_{\pi N}\left(q^{2}, q^{2}\right)=\frac{i}{q^{2}-m_{\pi}^{2}}\left(\frac{i \sigma}{f_{\pi}^{2}}+\frac{2 i}{f_{\pi}^{2}}\left(\omega \omega^{\prime} c_{2}+q \cdot q^{\prime} c_{3}\right)\right) \frac{i}{q^{2}-m_{\pi}^{2}}
$$




$$
+\left(-\frac{\sigma}{f_{\pi}^{2} m_{\pi}^{2}}\right) \frac{i}{q^{\prime 2}-m_{\pi}^{2}}+\frac{i}{q^{2}-m_{\pi}^{2}}\left(-\frac{\sigma}{f_{\pi}^{2} m_{\pi}^{2}}\right) .
$$

Amputating external pion legs by multiplying with $i\left(q^{2}-m_{\pi}^{2}\right)\left(q^{2}-m_{\pi}^{2}\right)$, we obtain the second term on the r.h.s. of amplitude (16). It agrees with the amplitudes (5, 6, 7), demonstrating explicitly that there is no inconsistency between chiral perturbation theory and PCAC.

One may be tempted to raise the objection that the amplitude (10) is inconsistent with the Ward identity (15), which is presumably a direct consequence of the underlying theory, QCD. However, it must be kept in mind that the Eq. (15) is a statement about isovector pseudoscalar currents, which are convention-dependent in contrast to vector or axialvector currents which have a physical realization, the electro-weak currents. In the language of PCAC, one arrives at the amplitudes (5, 6, 7) by identifying the pion interpolating field with the divergence of the axial-current, Eq.(4). In the formulation of Manohar and Georgi[2] this identification is not made, as is evidenced by Eq.(10). This difference is of no consequence for the evaluation of S-matrix elements 12, nor in the evaluation the effective mass ( Section 3 ).

We close this section with a few additional comments on the relation between the two formulations outlined above. First, we remark that although the second of the approaches described above is based on functional integrals, while the first is not, this is purely a notational distinction. We could just as well have derived the amplitude (10), in a functional integral formalism, using the action $S=\int d^{4} x\left(\mathcal{L}_{\mathrm{MG}}+\pi^{a} J^{a}\right)$, by taking derivatives of the corresponding generating functional with respect to $J^{a}$. The two approaches differ in the structure and coupling of their external sources. Second, there are complications arising at the one-loop level. At the one-loop level, in computing four-point and higher Green functions of the basic fields in models such as the non-linear sigma model, one encounters divergences off-mass-shell that cannot be removed - in a standard way - by chiral invariant counterterms. These divergences vanish on-mass-shell and may, more generally, be removed by a suitable redefinition of fields which have to be reexpressed by a non-linear combination of the original fields including space-time derivatives [24]. Introducing external fields via covariant derivatives in a chiral invariant manner in the generating functional framework, one obtains n-point Green functions involving currents, and chiral invariance is directly 
obtained to a given order in the loop expansion [25, 13]. In other words, this scheme generates by construction counter terms which are non-linear in the external sources (or contain space-time derivatives thereof) and which one otherwise would have to provide by hand in order to redefine the original fields in the first scheme. The point is once more: in the second scheme one has a relationship (dictated by the local chiral symmetry) between the usual terms in the lagrangian and the structures of the sources. By a redefinition (wave function renormalization) of the pion field one can make the source structures trivial, however, at the expense of a rather complicated non-linear field redefinition for the pion field which involves derivatives as the pseudo scalar source involved derivatives. There is of course - also in the second scheme - an arbitrariness in introducing the sources on the QCD-level such that chiral symmetry is locally preserved. But once this has been fixed, there is no arbitrariness any longer in the transcription down from the QCD level to the hadronic one. In this line of thought, the first scheme (especially at higher loop order) would correspond to a non-standard and rather complicated coupling of the external sources to the current quarks at the QCD level.

In the present work, we concentrate on tree level calculations, Green functions or correlators with at most two external pion legs, and the above complications do not arise. Further work is needed to clarify the situation at one-loop order, but a recent calculation[26] indicates that the two approaches discussed here also give the same effective meson mass to one-loop order, as the pion field redefinitions only contribute at higher order than $\mathcal{O}\left(\pi^{2}\right)$.

\section{The Effective Meson Mass in Nuclear Matter}

Given the problems encountered in extending chiral perturbation theory from the meson to the baryon sector, it is not surprising that a rigorous formulation of the expansion in nuclear matter has not yet been found. The presence of an additional scale, the Fermi momentum of nucleons, the breaking of Lorentz invariance, and nuclear correlations, add new levels of complexity to the formulation of a chiral expansion. As a first step, one simply uses a free space chiral expansion, such as those outlined above and evaluates nucleon operators at the mean field level, and 
consequently works with the lagrangian to linear order in density. One may again raise the question of the role of the pion interpolating field in this context. In what follows, we will argue that the basic idea, established rigourously in the case of free space scattering, that physically relevant observables are independent of the choice of field variables, also holds in nuclear matter. In this case, the relevant observable is the position of the pole of the pion propagator in symmetric nuclear matter 27, 4. The pole position is often referred to as the effective mass, which we shall also do here. In the absence of a general proof that the poles of the propagator are independent of the choice of field variables, we demonstrate that the two lagrangians discussed above, and most often employed in chiral perturbation theory, do indeed give the same value for the effective pion in mass in nuclear matter.

As alluded to above, in the nucleon mean field approximation we set $\langle\bar{N} N\rangle=\rho$ ( we also approximate the vector density by the scalar density. ) From the ManoharGeorgi lagrangian (8), we thereby obtain the inverse propagator for charged pions

$$
\begin{aligned}
D^{-1}(\omega, \mathbf{k}, \rho) & =\omega^{2}-\mathbf{k}^{2}-m_{\pi}^{2}-\Pi(\omega, \mathbf{k}, \rho) \\
\Pi(\omega, \mathbf{k}, \rho) & =-\frac{\rho}{f_{\pi}^{2}}\left(2\left(c_{2}+c_{3}\right) \omega^{2}-2 c_{3} \mathbf{k}^{2}+\sigma\right)+\mathcal{O}\left(m_{\pi}^{3}\right) .
\end{aligned}
$$

Evaluating the poles of the propagator we find the effective pion mass $m_{\pi}^{* 2}(\rho):=$ $\omega^{2}(\mathbf{k}=0 ; \rho)$ in symmetric nuclear matter to be

$$
\begin{aligned}
m_{\pi}^{* 2}(\rho) & =m_{\pi}^{2} \frac{1-\frac{1}{f_{\pi}^{2} m_{\pi}^{2}} \sigma \rho}{1+\frac{2}{f_{\pi}^{2}}\left(c_{2}+c_{3}\right) \rho}+\mathcal{O}\left(m_{\pi}^{3}\right) \\
& =m_{\pi}^{2}\left(1-\frac{\rho}{f_{\pi}^{2} m_{\pi}^{2}}\left(2\left(c_{2}+c_{3}\right) m_{\pi}^{2}+\sigma\right)\right)+\mathcal{O}\left(m_{\pi}^{3} ; \rho^{2}\right),
\end{aligned}
$$

where in Eq. (21) we explicitly show the prediction to linear order in density. Eq. (21) gives $m_{\pi}^{*}(\rho)=m_{\pi}-2 \pi a_{\pi N}^{+} \rho / m_{R}$, to linear order in density, where $m_{R}$ is the reduced mass of the pion-nucleon system. The behaviour of the effective mass to linear order in density is as expected from the lowest order optical potential, without reference to chiral perturbation theory. The effective mass receives additional contributions over those given by Eq.(20) at higher than linear order in density, from factors such as those mentioned at the beginning of this section. In the equations above, and in those which follow, we exhibit explicitly only the density dependence following from the chiral lagrangian, to $\mathcal{O}\left(Q^{2}\right)$, and in the mean-field approximation. 
Similarly, we consider the lagrangian $\mathcal{L}_{\mathrm{GSS}}$, Eq.(14), in the mean field approximation. Note that from (11) it is immediately apparent that since $U$, and therefore $\pi$, is a dummy variable, any observable must be independent of this field. In what follows, we obtain the effective pion mass in symmetric nuclear matter by two ( equivalent ) methods, the first uses the effective action $\Gamma$, ( see e.g. Ref.28 ), and the second, Feynman diagrams. In obtaining the effective action, the so-called classical pion field $\phi_{\pi}$ is defined with the help of the the generating functional in the nucleon mean field approximation, $Z[j, \rho]:=Z_{\mathrm{eff}}[\mathcal{M}, j, 0,0,0,0]$, as follows:

$$
\frac{\delta Z[j, \rho]}{\delta j^{a}}=\phi_{\pi}^{a}
$$

At tree level, we have

$$
Z[j, \rho]=S[\pi]+\int d^{4} x j^{a}(x) \pi^{a}(x)\left(1-\frac{\sigma \rho}{f_{\pi}^{2} m_{\pi}^{2}}\right),
$$

where the action $S$ is given by the lagrangian $\mathcal{L}_{\text {GSS }}$ in the nucleon mean field approximation, without the source $j^{a}$, namely, $S=\int d^{4} x \mathcal{L}^{(2)}(\rho)$ with

$$
\mathcal{L}^{(2)}(\rho)=\frac{f_{\pi}^{2}}{4}\left(g^{\mu \nu}+\frac{D^{\mu \nu} \rho}{f_{\pi}^{2}}\right) \operatorname{Tr}\left(\partial_{\mu} U \partial_{\nu} U^{\dagger}\right)+\frac{f_{\pi}^{2}}{4}\left(1-\frac{\sigma \rho}{f_{\pi}^{2} m_{\pi}^{2}}\right) \operatorname{Tr}\left(U^{\dagger} \chi+\chi^{\dagger} U\right),
$$

and $D^{\mu \nu} \equiv 2 c_{2} v^{\mu} v^{\nu}+2 c_{3} g^{\mu \nu}$, as follows from Eqs.(12) and (13). From Eq.(22), we obtain

$$
\phi_{\pi}^{a}=\left(1-\frac{\sigma \rho}{f_{\pi}^{2} m_{\pi}^{2}}\right) \pi^{a}
$$

The tree-level effective action, $\Gamma\left[\phi_{\pi}, \rho\right]=Z\left[\phi_{\pi}, \rho\right]-\int d^{4} x j \phi_{\pi}=S\left[\left(1-\frac{\sigma \rho}{f_{\pi}^{2} m_{\pi}^{2}}\right)^{-1} \phi_{\pi}\right]$, therefore reads

$$
\begin{aligned}
& \Gamma\left[\phi_{\pi}, \rho\right]= \\
& \frac{1}{2} \int d^{4} x\left(1-\frac{\sigma \rho}{f_{\pi}^{2} m_{\pi}^{2}}\right)^{-2}\left(\partial_{\mu} \phi_{\pi} \partial_{\nu} \phi_{\pi}\left(g^{\mu \nu}+\frac{D^{\mu \nu} \rho}{f_{\pi}^{2}}\right)-m_{\pi}^{2}\left(1-\frac{\sigma \rho}{f_{\pi}^{2} m_{\pi}^{2}}\right) \phi_{\pi}^{2}\right),
\end{aligned}
$$

from which we obtain the in-medium charged pion propagator

$$
D(q, \rho)=\frac{i\left(1-\frac{\sigma \rho}{f_{\pi}^{2} m_{\pi}^{2}}\right)^{2}}{q^{2}-m_{\pi}^{2}+\frac{\rho}{f_{\pi}^{2}}\left(\sigma+2 c_{2}(v \cdot q)^{2}+2 c_{3} q^{2}\right)}+\mathcal{O}\left(m_{\pi}^{3}\right) .
$$

As with the example of pion-nucleon scattering amplitudes considered earlier, it is instructive to rederive these results with the help of Feynman diagrams. From the 
lagrangian (14) in the mean field approximation, we obtain two vertices, $\odot$ and $\otimes$, connecting to two bare pion lines and one bare pion line, respectively. The vertices are

$$
\odot=i \frac{\rho}{f_{\pi}^{2}}\left(\sigma+2 c_{2}(v \cdot q)^{2}+2 c_{3} q^{2}\right) \quad \text { and } \quad \otimes=-\frac{\sigma \rho}{f_{\pi}^{2} m_{\pi}^{2}} .
$$

The in-medium pion propagator follows from summing all possible contributions dressing the bare pion propagator, as shown in the first line of Fig. 2a). The contributions involving $\odot$ yield a geometric series whose sum is denoted by the double dashed line in Fig. 2b). This yields the four diagrams of the second line of Fig. 2a), which factorize to yield the single expression (27).

The poles of the pion propagator in symmetric nuclear matter, (27), obtained from the lagrangian $\mathcal{L}_{\mathrm{GSS}}$, are identical to those of the propagator (19), following from the lagrangian $\mathcal{L}_{\mathrm{MG}}$. In both cases, the effective pion mass is given by Eq.(20). The fact that the lagrangian $\mathcal{L}_{\mathrm{GSS}}$ emodies the PCAC choice of pion interpolating field appears in the residue of the pion propagator, not in the effective pion mass. We therefore conclude that there is no discrepancy in the effective mass obtained from theories using different interpolating pion fields 10, 11. It is furthermore to be noted that, within the approximations that we are working with and have stated above, the effective mass predicted by both models is identical to all orders in nuclear density. From the the tree-level calculation of the effective pion mass, one cannot conclude that the two approaches are intrinsically different, to higher than linear order in density 11.

To conclude this section, we discuss the relation of the effective pion mass in symmetric nuclear matter, to the Gell-Mann-Oakes-Renner ( GMOR ) relation 14. The Gell-Mann-Oakes-Renner ( GMOR ) relation reads

$$
f_{\pi}^{2} m_{\pi}^{2}=-\frac{m_{u}+m_{d}}{2}\langle 0|\bar{u} u+\bar{d} d| 0\rangle+\mathcal{O}\left(m_{\pi}^{4}\right)
$$

The GMOR relation is derived from the effective second-order lagrangian $\mathcal{L}_{\pi \pi}^{(2)}$, Eq.(12), by the identification $Z_{\mathrm{QCD}}=Z_{\text {eff }}$, which leads to

$$
\left.\frac{\delta Z_{\mathrm{QCD}}}{\delta \mathcal{M}(x)}\right|_{\mathcal{M}=0}=-\langle 0|\bar{u} u+\bar{d} d| 0\rangle=2 f_{\pi}^{2} B
$$

Solving for $B$ and using the relation $B\left(m_{u}+m_{d}\right)=m_{\pi}^{2}$ one thus obtains Eq.(29) 13. To study the GMOR relation with respect to the in-medium pion mass, we use the 
lagrangian $\mathcal{L}_{\mathrm{GSS}}$ in the nucleon mean field approximation, Eq.(24). Since matter breaks Lorentz invariance (but still keeps rotational invariance, if it is isotropic), it is convenient to separate space and time components [29 via ( see also Ref. 30]),

$$
f_{\pi}^{2}\left(g^{\mu \nu}+\frac{D^{\mu \nu} \rho}{f_{\pi}^{2}}\right)=f_{t}^{\star 2}(\rho) g^{00}+f_{s}^{\star 2}(\rho) g^{i i}
$$

where the time-component is given by

$$
f_{t}^{\star 2}(\rho)=f_{\pi}^{2}\left(1+\frac{D^{00} \rho}{f_{\pi}^{2}}\right)+\mathcal{O}\left(m_{\pi}\right) .
$$

Starting from Eq.(24) and using the same method as to derive Eq.(30), we obtain the density dependent quark condensate

$$
\langle\bar{u} u+\bar{d} d\rangle_{\rho}=\langle 0|\bar{u} u+\bar{d} d| 0\rangle\left(1-\frac{\sigma \rho}{f_{\pi}^{2} m_{\pi}^{2}}\right)+\mathcal{O}\left(m_{\pi}\right),
$$

a result which is, in fact, model-independent 31]. Eq.(33), when combined with the effective pion mass as given in Eq.(20), yields the in-medium GMOR relation in the nucleon mean-field approximation:

$$
f_{t}^{\star 2}(\rho) m_{\pi}^{\star 2}(\rho)=-\frac{m_{u}+m_{d}}{2}\langle\bar{u} u+\bar{d} d\rangle_{\rho}+\mathcal{O}\left(m_{\pi}^{3}\right)
$$

( Other discussions of the GMOR relation at finite density are given in Ref.[32]. ) It is therefore only the time-component of the coupling constant, $f_{t}^{\star}$ that enters in the GMOR relation at finite density. As a function of density, $f_{t}^{\star}$ decreases, $m_{\pi}^{\star}$ increases, though very slowly, and $-\langle\bar{u} u+\bar{d} d\rangle_{\rho}$ decreases.

It is worthwhile to check that $f_{t}^{\star}(\rho)$, as given by Eq.(32), agrees with the definition in terms of the axial current coupling to the pion in matter,

$$
\left\langle 0\left|\bar{q} \gamma_{0} \gamma_{5} \frac{1}{2} \tau^{a} q\right| \pi^{b}\right\rangle_{\rho}=i p_{0} \delta^{a b} f_{t}^{\star}(\rho)+\mathcal{O}\left(m_{\pi}^{3}\right)
$$

The expectation value may be evaluated from the axial vector two point function, which reads, at zero nucleon density, (see Ref.[13])

$$
\begin{aligned}
\left.\frac{\delta^{2} Z_{\mathrm{eff}}}{\delta a_{\mu}^{a}(-q) \delta a_{\nu}^{b}(q)}\right|_{a=v=p=0 ; s=\mathcal{M}} & =i \int d x e^{i q(x-y)}\left\langle 0\left|T A_{\mu}^{a}(x) A_{\nu}^{b}(y)\right| 0\right\rangle \\
& =\delta^{a b}\left\{g_{\mu \nu} f_{\pi}^{2}+\frac{q_{\mu} q_{\nu} f_{\pi}^{2}}{m_{\pi}^{2}-q^{2}}\right\}+\mathcal{O}\left(q^{2}\right) .
\end{aligned}
$$


Eq.(36), and other correlators, may be evaluated at finite density, in the mean field approximation, by reinstating the general sources $s, v^{\mu}$ and $a^{\mu}$ in $Z_{\text {eff }}$, expanding the action to second order in the pionic field, and integrating out the pionic degrees of freedom. The second order variation with respect to the external sources ( e.g. $a_{0}^{a}$ for the case in Eq.(36) ) then gives the two-point function. For example, one finds that up to $\mathcal{O}\left(m_{\pi}\right)$ corrections - the time-component of the axialvector correlator in matter has the same form as Eq.(36), but with $f_{\pi}^{2}$ replaced by $f_{t}^{* 2}(\rho)$, Eq.(32), and $m_{\pi}$ replaced by the effective mass (20). Thus the desired equivalence is established. This result is independent of the off-shell extension of the pion field, as the pseudoscalar sources, $p^{a}$, do not enter in this calculation.

In an analogous manner one may arrive at other relations valid at finite density. Evaluation of the in-medium pseudoscalar correlator results in (see Eq.(17))

$$
g_{\pi}^{\star 2}(\rho)=\left(2 B f_{\pi}\right)^{2} \frac{\left(1-\frac{\sigma \rho}{f_{\pi}^{2} m_{\pi}^{2}}\right)^{2}}{1+\frac{D^{00} \rho}{f_{\pi}^{2}}}+\mathcal{O}\left(m_{\pi}\right) .
$$

This result is dependent on the off-shell extension of the pion field, as the calculation explicitly involves functional derivatives with respect to the pseudoscalar source, $p^{a}$, and holds therefore only in the second scheme, compatible with PCAC. The axialvector-pseudoscalar correlator is scheme-dependent, as well. Using Eqs.(33) and $(20)$ one can then check that the finite-density version of the PCAC relation (17) holds in the second scheme:

$$
f_{t}^{\star}(\rho) m_{\pi}^{\star 2}(\rho)=\frac{m_{u}+m_{d}}{2} g_{\pi}^{\star}(\rho)+\mathcal{O}\left(m_{\pi}^{3}\right)
$$

A caveat is the rather rapid decrease of the in-medium quark condensate, Eq.(33), ( which drops at nuclear matter density already to about two thirds of its vacuum value ), as a large value of the quark condensate is a precondition on counting the current quark mass matrix as $\mathcal{O}\left(Q^{2}\right)$. If the (in-medium) quark condensate gets smaller and smaller, it is not obvious that the (in-medium) four-quark condensate can be safely neglected at $\mathcal{O}\left(Q^{2}\right)$. It may become as important as the two-quark condensate, such that the quark mass matrix should rather be counted as $\mathcal{O}\left(Q^{1}\right)$ ( see Ref. 33] in a different context ). Note that even in symmetric nuclear matter all quantities of $\mathcal{O}\left(Q^{2}\right)$ get their first corrections already at $\mathcal{O}\left(Q^{3}\right)$ - as the meanfield calculation showed - and not at $\mathcal{O}\left(Q^{4}\right)$ as their free-space analogs. One might 
speculate that this fact is already a signal or precursor of a change in the relation between $m_{\pi}^{\star 2}(\rho)$ and the current quark mass matrix $\mathcal{M}$ from that given by the vacuum GMOR relation. This phenomenon has eventually to take place with increasing density, as at chiral restoration ( or perhaps even earlier ) the pion should lose its Goldstone character and approximate the behavior of a "normal" meson with the corresponding relation between its (effective) mass and the current quark masses: $m_{\pi}^{*} \propto \hat{m}=\left(m_{u}+m_{d}\right) / 2$ [34]. Thus the in-medium GMOR relation (34) is likely to break down at large nuclear densities.

To summarize the results of this Letter, since the isospin even scattering length is $a_{\pi N}^{+}=-0.01 m_{\pi}^{2}$, the pion mass increases slightly to linear order in density in symmetric nuclear matter [9]. This result is valid at low density, and is, of course, expected, whether one uses a chiral effective lagrangian or not. Tree-level chiral perturbation theory in the nucleon mean field approximation predicts that the effective mass continues to grow with increasing density, as given by Eq.(20). Eq.(20) was derived from two formulations of chiral perturbation theory. One of these 13, 18, 20 corresponds to adopting the PCAC choice of pion interpolating field, while the other 22 does not. The fact that the two formulation give identical results indicates that the effective mass in matter is generally independent of the choice of pion field variables adopted in the effective lagrangian.

To which extent these predictions are correct can only finally be decided by better knowledge of higher density terms, both in terms of nuclear correlations, and at the level of the lagrangian. Confining the discussion to the chiral lagrangian, at tree-level, and in the nucleon mean field approximation, we have found that the two approaches outlined above give identical effective pion mass to all orders in nuclear density. This seriously questions the claim that differences in the choice of pion field manifest themselves as different predictions to second order in density and above 11.

A number of additional factors must be taken into consideration in studying kaon condensation in neutron star matter, such as the additional attraction mediated by the exchange of the $\rho$ and $\omega$ vector mesons, i.e. the Weinberg term. Other pertinent issues include the role of resonances such as the $\Lambda(1405)$, which governs low-energy $K^{-} p$ scattering and the coupling to the $\Sigma \pi$ channel [10, 35], the role of hyperons 1 , 36], the effect of $\mathcal{O}\left(Q^{3}\right)$ contributions in chiral perturbation theory [6] and nuclear 
correlations [6, 7]. These complications do not detract from our basic conclusion, namely that the effective kaon mass, in neutron star matter, is independent of the choice of kaonic field varmade in the effective lagrangian. Although kaon-nucleon scattering amplitudes, obtained from a specific choice of kaon interpolating field, may be repulsive off meson-mass-shell, such as the PCAC amplitude in the Weinberg point, this does not imply that that the effective kaon mass tends to increase in matter, precluding kaon condensation. In this respect, the effective kaon mass in matter, as obtained originally by Kaplan and Nelson[1], and in most studies that have followed, has been correctly evaluated.

\section{Acknowledgements}

We are grateful to F. Beck, M. Kirchbach, M. Lutz, and W. Weise for discussions and to H. Bijnens, G. E. Brown, N. Kaiser, A. Manohar, U.-G. Meißner, M. Rho and I. Zahed for discussions and a critical reading of a previous version of the manuscript. We thank the ECT*, Trento, Italy, for support to attend its International Workshop

on Chiral Symmetry in Hadrons and Nuclei. A. W. would like to thank NORDITA and the Niels Bohr Institute for support and hospitality during his stays in Copenhagen where part of this work was carried out. 


\section{References}

[1] D. B. Kaplan and A. E. Nelson, Phys. Lett. B175 (1986) 57.

[2] A. Manohar and H. Georgi, Nucl. Phys. B234 (1984) 189; H. Georgi, Weak Interactions and Modern Particle Theory ( Benjamin/Cummings, Menlo Park, 1984 ).

[3] G. E. Brown, K. Kubodera, M. Rho and V. Thorsson, Phys. Lett. B291 (1992) 355.

[4] V. Thorsson, M. Prakash and J. M. Lattimer, Nucl. Phys. A572 (1994) 693; A574 (1994) 851.

[5] G. E. Brown, C.-H. Lee, M. Rho and V. Thorsson, Nucl. Phys. A567 (1993) 937.

[6] C.-H. Lee, H. Jung, D.-P. Min and M. Rho, Phys. Lett. B326 (1994) 14; C.-H. Lee, G. E. Brown and M. Rho, SNUTP-94-28, hep-ph/9403339; C.-H. Lee, G. E. Brown, D.-P. Min and M. Rho, SNUTP-94-50, hep-ph/9406311.

[7] V. R. Pandharipande, C. J. Pethick and V. Thorsson, work in progress.

[8] G. E. Brown, V. Koch and M. Rho, Nucl. Phys. A535 (1991) 701.

[9] J. Delorme, M. Ericson and T.E.O. Ericson, Phys. Lett. B291 (1992) 379.

[10] H. Yabu, S. Nakamura, F. Myhrer and K. Kubodera, Phys. Lett. B315 (1993) 17; H. Yabu, S. Nakamura and K. Kubodera, Phys. Lett. B317 (1993) 269.

[11] H. Yabu, F. Myhrer and K. Kubodera, Phys. Rev. D50 (1994) 3549.

[12] S. Coleman, J. Wess and B. Zumino, Phys. Rev. 177 (1969) 2239.

[13] J. Gasser and H. Leutwyler, Ann. Phys. (N.Y.) 158 (1984) 142; Nucl. Phys. B307 (1988) 763; (1991) 353.

[14] M. Gell-Mann, R. J. Oakes and B. Renner, Phys. Rev. 175 (1968) 2195. 
[15] G. Höhler, Landolt-Börnstein, Vol. I/9b2, ed. H. Schopper ( Springer, Berlin, $1983)$.

[16] T. P. Cheng and R. F. Dashen, Phys. Rev. Lett. 26 (1971) 594.

[17] See S. L. Adler and R. F. Dashen, Current algebra and applications to particle physics ( Benjamin, New York, 1968 ).

[18] J. Gasser, M. E. Sainio and A. S̆varc, Nucl. Phys. B307 (1988) 779.

[19] E. Jenkins and A. Manohar, Phys. Lett. B255 (1991) 558.

[20] V. Bernard, N. Kaiser and U.-G. Meißner, Phys. Lett. B309 (1993) 421.

[21] J. Gasser, H. Leutwyler and M. E. Sainio, Phys. Lett. B253 (1991) 252.

[22] R. Koch, Nucl. Phys. A448 (1986) 707.

[23] V. Bernard, N. Kaiser, J. Kambor and U.-G. Meißner, Nucl. Phys. B388 (1992) 315.

[24] T. Appelquist and C. Bernard, Phys. Rev. D23 (1981) 425.

[25] J. Honerkamp, Nucl. Phys. B36 (1972) 130.

[26] C.-H. Lee and M. Rho, private communication.

[27] G. Baym and E. Flowers, Nucl. Phys. A222, (1974) 29; A. E. Nelson and D. B. Kaplan, Phys. Lett. B192 (1987) 193; T. Muto and T. Tatsumi, Phys. Lett. B283 (1992) 165.

[28] S. Pokorski, Gauge Field Theories ( Cambridge University Press, 1987 ).

[29] M. Kirchbach and D. O. Riska, Nucl. Phys. A578 (1994) 511.

[30] H. Leutwyler, Phys. Rev D49 (1994) 3033.

[31] E. G. Drukarev and E. M. Levin, Nucl. Phys. A511 (1988) 697; T. D. Cohen, R. J. Furnstahl and D. K. Griegel, Phys. Rev. Lett. 67 (1991); Phys. Rev C45 (1992) 1881; M. C. Birse, J. Phys. G. 20 (1994) 1537. 
[32] V. Bernard and U.-G. Meißner Nucl. Phys. A489 (1988) 647; M. Lutz, A. Steiner and W. Weise, Nucl. Phys. A542 (1992) 521; A574 (1994) 755.

[33] M. Knecht and J. Stern, IPNO-TH-94-53, to be published in the second edition of the DAPHNE physics handbook, Eds. L. Maini, G. Pancheri and N. Paver, hep-ph/9411253.

[34] A. Wirzba and V. Thorsson, to be published in the proceedings of the workshop Hirschegg '95: Hadrons in Nuclear Matter.

[35] M. J. Savage, Phys. Lett. B331 (1994) 411; J. Schaffner, A. Gal, I. N. Mishustin, H. Stöcker, and W. Greiner, Phys. Lett. B334 (1994) 268; V. Koch, Phys. Lett. B377 (1994) 7; A. Steiner and W. Weise, to be published.

[36] T. Muto, Prog. Theor. Phys. 89 (1993) 415; P. J. Ellis, R. Knorren and M. Prakash, in progress. 


\section{Figure Captions}

Fig. 1 Feynman diagrams for the connected 4-point $\pi \pi \bar{N} N$ Green function $A_{\pi N}\left(q^{2}, q^{2}\right)$.

a) Diagrams with two external pion legs. b) and c) Diagrams with one external pion leg. The full lines represent the nucleon legs, the dashed lines the pion legs and the double lines the external pseudo-scalar sources $j^{a}$.

Fig. 2 The in-medium pion propagator. The dashed line represents a free pion, and the vertices $\odot$ and $\otimes$ are given in Eq.(28). a) The sum of all contributions.

b) The sum of the geometric series involving $\odot$ contributions. 


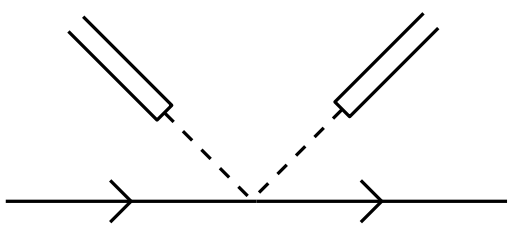

(a)

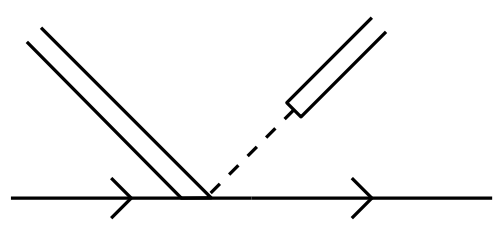

(b)

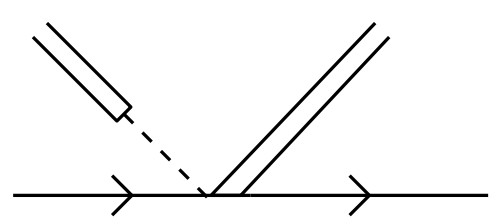

(c) 


$$
\begin{aligned}
& \ldots-\ldots+-\ldots+-\ldots+\ldots+\cdots+\cdots
\end{aligned}
$$

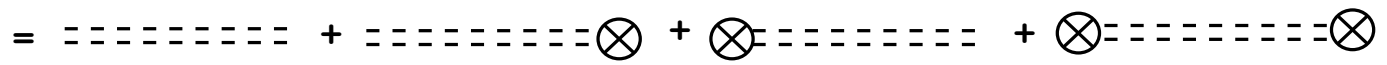

(a)

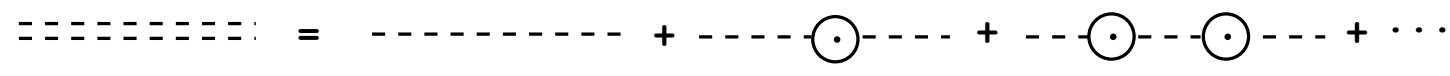

(b) 